\title{
Cooperação de Veículos Baseada em Características Sociais para Disseminação de Dados de Eventos Críticos Urbanos
}

\author{
Alisson Yury ${ }^{1}$, Everaldo Andrade ${ }^{1}$, Aldri Santos ${ }^{2}$, Fernando Matos $^{1}$ \\ ${ }^{1}$ Centro de Informática - Universidade Federal da Paraíba (UFPB) \\ ${ }^{2}$ Departamento de Informática - Universidade Federal do Paraná (UFPR) \\ alisson.yury@cc.ci.ufpb.br, everaldo.andrade@ppgi.ci.ufpb.br, \\ aldri@inf.ufpr.br, fernando@ci.ufpb.br
}

\begin{abstract}
Critical urban events need to be efficiently handled, for instance, through rapid notification. Vehicular Ad Hoc Networks (VANETs) are a promising choice in supporting notification of information on arbitrary critical events. Although the dynamicity of VANETs compromises the dissemination process, the connections among vehicles based on the users' social interests allow for optimizing message exchange and data dissemination. This paper introduces SOCIABLE, a robust data dissemination system for critical urban events that operates in a SIoV network. It is based on vehicles' community with common interests and/or similar routines and employs social influence of vehicles according to their network location to select relay vehicles. In a comparative analysis on NS3 with the MINUET system, SOCIABLE achieved $36.56 \%$ less messages in a dense VANET and a maximum delay of 0.028s in a sparse VANET, delivering critical event data in a real-time and robust way without overloading the network.
\end{abstract}

Resumo. Eventos críticos urbanos precisam ser alertados eficientemente, por exemplo, através de uma notificação rápida. As redes veiculares (VANETs) são uma potencial alternativa, para apoiar a notificação das informações dos eventos críticos arbitrários, devido à sua ubiquidade nas cidades. Embora a dinamicidade das VANETs comprometa o processo de disseminação, as conexões entre veículos a partir dos interesses sociais dos usuários oferecem um modo para otimizar a troca de mensagens e a disseminação de dados. Este artigo propõe SOCIABLE, um sistema de disseminação robusta de dados de eventos urbanos críticos que atua numa rede SIoV. Ele baseia-se em comunidade de veículos com interesses comuns elou rotinas similares e na influência social dos veículos pela sua posição na rede, para a escolha dos veículos retransmissores. Numa análise comparativa no NS3 com o sistema MINUET, o SOCIABLE gerou menos 36,56\% de mensagens transmitidas numa VANET densa e um pico máximo de atraso de 0,028s numa VANET esparsa, entregando dados de eventos críticos em tempo real e de maneira robusta sem sobrecarregar a rede.

\section{Introdução}

Um dos grandes desafios dos centros urbanos consiste no tratamento de eventos críticos que muitas vezes ocorrem de forma aleatória no tempo e no espaço. Eventos críticos são aqueles que, se não tratados eficientemente, podem impactar no bom andamento da cidade 
(e.g. buracos nas vias, engarrafamentos, etc) e/ou colocar em risco a integridade das pessoas (incêndios, acidentes, etc) [Monreal 2018]. A infraestrutura ubíqua suportada pelas Tecnologias da Informação e Comunicação - TICs (redes de comunicação, sensores, atuadores, etc), desempenha um papel primordial como facilitador na detecção e disseminação dos eventos urbanos [Andrade et al. 2019]. As VANETs tornam-se uma opção natural a esta tarefa devido à ubiquidade dos veículos. Assim, a movimentação dos veículos que trafegam nas vias urbanas pode auxiliar na detecção de eventos ao portar câmeras e sensores embarcados. Além disso, as conectividades entre veículos (V2V) e a infraestrutura de redes (V2I), colaboram para disseminar informações até uma entidade externa responsável por tratar o evento. Contudo, a intermitência das conexões em razão da alta dinamicidade topológica das VANETs dificulta a transmissão de informações, tal que soluções de disseminação dependam quase exclusivamente de conexões oportunísticas [Vegni and Loscrí 2015]. Além disso, veículos podem não ter interesse em cooperar na disseminação, criando comportamento egoísta na rede. Logo, essas questões são desafios ao tratamento de eventos porque sua criticidade exige uma disseminação robusta [Khan and Franzle 2015], tal que a informação chegue rápida e correta à entidade externa.

Baseado nos conceitos de Cyber Physical-Systems (CPS) e Cyber-Human-Systems (CHS), tem-se buscado empregar novas formas de construir e coordenar os sistemas em redes como meio de obter uma gerência autônoma mais inteligente. Dentre estes, os conceitos sociais vêm ganhando força por permitir a criação de relacionamentos entre os dispositivos a partir dos seus usuários. A Social Internet of Things (SIoT) define cinco tipos de relacionamentos: (i) Parental (POR), relação estabelecida entre dispositivos produzidos pelo mesmo fabricante; (ii) Co-work (C-WOR), relação entre objetos cooperando em uma mesma aplicação IoT; (iii) Co-location (C-LOR), relacionamento entre objetos que são usados no mesmo local; (iv) Ownership (OOR), relação entre os dispositivos de um mesmo usuário; e o Social Objects Relationship (SOR), relação estabelecida entre objetos que entram em contato, de maneira esporádica ou contínua. A aplicação da SIoT ao contexto de VANETs originou a Social Internet of Vehicles (SIoV) como uma alternativa ao compartilhamento de mensagens entre veículos que possuem algum tipo de relacionamento. Na SIoV, os relacionamentos normalmente são estabelecidos de acordo com o comportamento dos motoristas. Assim, os veículos podem socializar para alcançar um objetivo em comum [Silva and Iqbal 2019]; por exemplo, motoristas que usam uma mesma avenida para se deslocarem num dado horário têm interesses em saber a existência de algum acidente nesta avenida, o que poderia causar atrasos.

O estabelecimento e a percepção de comunidade são duas técnicas de redes sociais empregadas em redes veiculares [Wang et al. 2019]. Apesar das comunidades formadas em VANETs não serem tão fortes quanto aquelas formadas em redes sociais tradicionais, é possível utilizar sua organização temporal/espacial para auxiliar na disseminação oportunística de dados referentes à eventos que ocorrem de maneira aleatória no tempo/espaço. Isto pode ser alcançado, por exemplo, através da inferência social dos veículos, ao estimar as comunidades através dos relacionamentos anteriormente formados pelos motoristas [Wu and Solmaz 2019]. Além disso, ao usar comunidades diminui-se a propensão por comportamentos egoístas dos veículos. Técnicas de Social Network Analysis (SNA) também auxiliam na identificação de componentes influentes de uma rede social a fim de melhorar a eficácia e a eficiência dos serviços de rede [Rahim et al. 2018b]. No contexto SIoV, a rede pode possuir um pequeno número de nós altamente influentes, que deter- 
minam a maior parte da conectividade e do fluxo de informação, podendo ser veículos que possuem vantagens de conexão sobre outros, e causem impacto na disseminação de informação, nos protocolos de roteamentos, nas medidas de segurança, dentre outros.

Embora existam trabalhos sobre como o comportamento humano afeta a interdependência social dos veículos na VANET, apenas alguns deles mostram como os veículos estabelecem relacionamentos sociais. Alguns utilizam métodos de crowdsourcing para prover vários serviços como sensoriamento [Xu et al. 2019] e gerência de mobilidade urbana [Akabane et al. 2019]. Outros trabalhos aplicam comunidades virtuais e técnicas de centralidade para selecionar dispositivos retransmissores para uma melhor disseminação dos dados [Cunha et al. 2014]. Para trabalhos que tratam eventos críticos urbanos apenas existe [Wang et al. 2018], porém ele foca na mobilidade urbana quando ocorre a detecção do evento crítico e não auxilia na entrega de dados ao tratamento do evento.

Este trabalho propõe um sistema que suporta a detecção e a disseminação robusta de eventos críticos urbanos ao considerar as características sociais dos dispositivos veiculares em VANETs. Este sistema, chamado SOCIABLE (SOCial monItoring and disseminAtion of urBan criticaL Events), leva em conta os relacionamentos de objetos sociais (SOR), esporádicos ou contínuos, estabelecidos entre os veículos que possuem interesses e/ou rotinas similares para a criação de comunidades locais. Comunidades e técnicas de centralidade apoiam uma disseminação direcionada (multicast) através da escolha de retransmissores, a fim de realizar uma entrega de dados mais ágil, aumentando a satisfação do usuário (Qualidade de Experiência - QoE), além de melhorar desempenho e robustez (Qualidade de Serviço - QoS) na disseminação dos eventos. Resultados de simulações no NS3 comprovam que o SOCIABLE realiza a disseminação dos dados do evento pela colaboração entre os veículos. Além disso, testes comparativos com uma solução que não emprega parâmetros sociais mostram que o SOCIABLE alcança resultados similares ou melhores com uma menor sobrecarga da rede.

O restante do artigo está estruturado como descrito a seguir. A Seção 2 apresenta os trabalhos relacionados. A Seção 3 descreve o SOCIABLE e o seu funcionamento. A Seção 4 apresenta uma avaliação comparativa do SOCIABLE e os resultados alcançados. Finalmente, a Seção 5 apresenta as considerações finais do trabalho.

\section{Trabalhos Relacionados}

Devido à ubiquidade de veículos na malha viária, várias soluções na literatura têm utilizados as VANETs para auxiliar no cotidiano das cidades ao tratar alguma questão urbana específica. Contudo, a maioria das soluções assume que os veículos participam e cooperam ativamente na disseminação, o que na realidade geralmente não acontece. Por exemplo, veículos com comportamento egoísta apenas cooperam na disseminação com aqueles que eles têm forte relacionamento social ou interesses em comum. Outras soluções dependem de uma entidade centralizada ou sobrecarregam a rede com mensagens duplicadas.

Em [Shrestha et al. 2018], os autores propõem uma solução para disseminação de mensagens de eventos críticos em VANETs com o apoio de um servidor na nuvem. Apesar de realizarem a disseminação, os veículos requisitam e recebem respostas do servidor para fazer a disseminação. Logo, a latência de comunicação com a nuvem aumenta o tempo de resposta, o que compromete a eficiência, principalmente ao considerarmos eventos críticos. Em [Yaqub et al. 2018], um esquema para disseminação de dados críticos 
em redes veiculares de dados nomeados propõe um algoritmo de recuperação de dados críticos para permitir os veículos obterem dados de múltiplos provedores na vizinhança do veículo usando um único pacote de interesse. Embora este esquema consiga gerenciar o encaminhamento de dados de maneira eficiente, em redes esparsas ele não consegue entregar muitos pacotes e o custo de transmissão de mensagens aumenta em razão do cache temporário para cada mensagem na rede. Já em [Andrade et al. 2019], os autores propõem um sistema chamado MINUET para o monitoramento e disseminação de eventos urbanos baseado em uma estratégia cooperativa de disseminação através de agrupamentos. Embora o MINUET seja eficaz, por utilizar estratégia de inundação, ele tem alta sobrecarga de mensagens duplicadas sendo transmitidas na rede.

Estudos têm investigado os benefícios do uso de métricas sociais para auxiliar na decisão de disseminação de dados em redes ad hocs, inclusive VANETs. Em [Cunha et al. 2014], as trocas de mensagens entre os veículos possibilitam descobrir o grau de cada veículo e seu coeficiente de agrupamento, auxiliando no encaminhamento de mensagens. Embora a solução garanta a entrega das mensagens, em ambientes de baixa densidade ela gera um grande custo de transmissão de mensagens devido ao armazenamento de mensagens. Já em [Campolo et al. 2018] um framework baseado em Software Defined Networking (SDN) auxilia no estabelecimento de relações sociais entre veículos e facilita a entrega de dados em comunicações Vehicle-to-Everything (V2X). Apesar de na disseminação de alertas o framework obter melhores resultados comparado ao uso da rede celular, ele depende de dispositivos na borda (switches) e de um servidor centralizado.

Outros trabalhos se preocupam com o comportamento egoísta dos dispositivos veiculares em redes. Em [Rahim et al. 2018b], são utilizadas técnicas sociais (grau de centralidade, o conhecimento da comunidade local e global, e a atividade social) e de reputação para conseguir a cooperação entre todos os veículos da rede e assegurar a retransmissão dos dados. Em redes esparsas, é possível que os veículos tenham baixa ou nenhuma atividade social. Além disso, caso não haja um veículo com uma melhor reputação que o veículo retransmissor atual, este armazena os dados até que consiga encontrar um nó com melhor reputação, o que aumenta o custo de transmissão das mensagens. Em [Wang et al. 2018] é proposto um sistema de gerenciamento do tráfego de uma cidade inteligente em situações de eventos anormais utilizando crowdsensing, onde uma técnica de incentivo monetário virtual encorajam os veículos a cooperarem com o encaminhamento de mensagens. Eles utilizaram a estrutura da rede de telefonia junto com as RSUs para um melhor custo-benefício. Entretanto, os veículos podem ajudar na disseminação e não serem recompensados, desperdiçando recursos.

\section{Sistema Cooperativo de Disseminação de Eventos Críticos Urbanos}

Esta seção detalha o sistema SOCIABLE (SOCial monItoring and disseminAtion of urBan criticaL Events), que trata de um sistema distribuído de monitoramento colaborativo e robusto de eventos críticos urbanos baseado em SIoV (Social Internet of Vehicles). Nós inicialmente apresentamos as características do ambiente urbano e sua infraestrutura onde o sistema SOCIABLE atua, bem como a sua arquitetura e componentes. Em seguida, ilustramos a operação do SOCIABLE na disseminação diante de um evento crítico. 


\subsection{Ambiente SIoV e Arquitetura do SOCIABLE}

O ambiente SIoV de operação do SOCIABLE consiste de dois níveis, como ilustra a Figura 1. O Nível Físico (NF) compreende os veículos que trafegam nas malhas viárias continuamente, e com diferentes velocidades. Assume-se que os veículos podem se comunicar com outros veículos, Veículos-a-Veículos (V2V), ou com a Infraestrutura Urbana através de Estações Base (EBs), Veículos-a-Infraestrutura (V2I), criando redes veiculares híbridas. Os eventos urbanos críticos (EUC) podem ocorrer de maneira aleatória no tempo e espaço. Um evento urbano crítico consiste de qualquer evento que possa impactar no dia-a-dia da cidade e dos seus cidadãos, como por exemplo, incêndios, acidentes, delitos, obstruções nas vias, dentre outros. Esses eventos devem ser notificados às Entidades Externas (EE), que representam autoridades competentes responsáveis pelo tratamento de eventos. As EEs se comunicam, através das EBs, com a rede veicular não-estruturada.

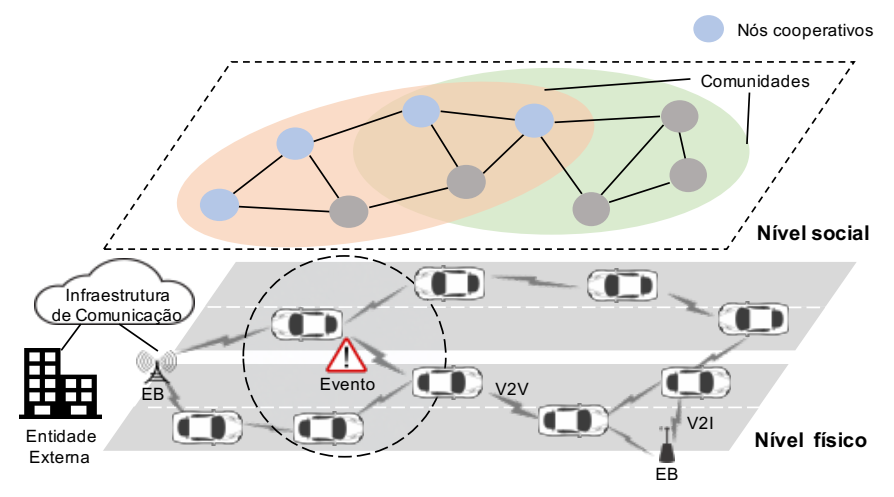

Figura 1. Modelo de SloV

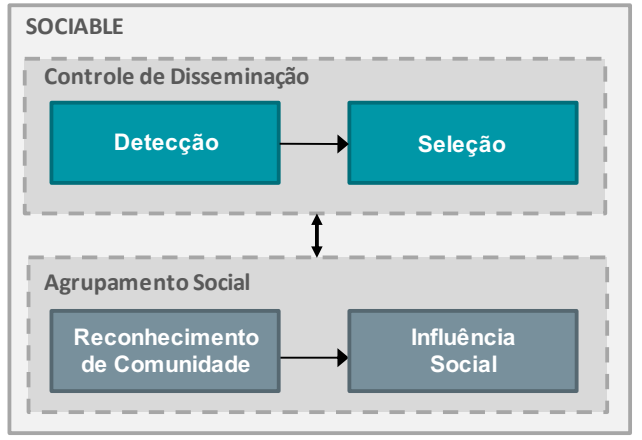

Figura 2. Arquitetura SOCIABLE

O Nível Social (NS) consiste dos perfis sociais dos veículos, que são construídos com base nas propriedades sociais (ex: rotina similar, interesse, local de trabalho) dos motoristas. Como toda rede social, os nós (veículos) da rede possuem relacionamentos que podem ser criados e desfeitos ao longo do tempo. Além disso, nós com interesses ou propriedades em comum podem fazer parte de uma comunidade. Por exemplo, nós que trafegam nas mesmas vias e nos mesmos horários (mesma rotina) podem fazer parte de uma comunidade para trocar mensagens. Um nó pode fazer parte de mais de uma comunidade. Nós que fazem parte de uma mesma comunidade já possuem uma prédisposição para colaborar. Assim, alguns (ou todos) nós de uma comunidade podem cooperar para realizar uma tarefa, como por exemplo, a disseminação de dados de um evento crítico.

Neste ambiente SIoV, veículos que detectam um evento podem iniciar uma cooperação com outros para colaborar na disseminação do evento, desde que eles façam parte da mesma comunidade. Assim, considere um conjunto $V$ de veículos $\left\{v_{1}, v_{2}, \ldots, v_{n}\right\}$ e um conjunto $E$ de eventos $\left\{e v_{1}, e v_{2}, \ldots, e v_{m}\right\}$, qualquer veículo $v_{i} \in V$ pode detectar um evento $e v_{j} \in E$, desde que $e v_{j}$ esteja no raio de alcance de $v_{i}$. Utilizando Social Objects Relationships (SOR), estabelecidos a partir da frequência com que veículos entram em contato de forma esporádica ou contínua, os veículos podem formar comunidades com outros veículos que compartilhem o mesmo interesse/perfil social. Logo, o ambiente SIoV também possui um conjunto $C$ de comunidades $\left\{c_{1}, c_{2}, \ldots, c_{p}\right\}$ em que para qualquer comunidade $c_{k} \in C, c_{k} \subseteq V$. Assim, um veículo $v_{i} \in c_{k}$ se, e somente 
se, $v_{i}$ possui o mesmo interesse/perfil social da comunidade $c_{k}$. Quando um evento $e v_{j}$ é detectado por um veículo $v_{i} \in c_{k}$, inicia-se a formação de um conjunto $D\left(e v_{j}\right) \subseteq c_{k}$, definido como um sub-conjunto de veículos da comunidade $c_{k}$ que cooperam na disseminação de $e v_{j}$. A arquitetura do SOCIABLE, conforme Figura 2, compreende o módulo Agrupamento Social, que gerencia as comunidades sociais formadas por veículos que possuem algum SOR; e o módulo Controle de Disseminação, que coordena a detecção dos eventos e a seleção dos veículos que cooperam na disseminação dos dados do eventos até às EEs.

O módulo Agrupamento Social coordena a criação e manutenção das comunidades sociais, e calcula a importância social que o veículo tem na comunidade. Este módulo possui dois componentes: Reconhecimento de Comunidade e Influência Social. O componente Reconhecimento de Comunidade gerencia o SOR entre os veículos através da identificação da comunidade local do veículo. A comunidade local é formada por veículos que fazem parte da mesma comunidade que o veículo atual e estão diretamente conectados a ele (vizinhos). Isto é importante porque apenas veículos da comunidade local cooperam para disseminar o evento. Todo $v \in V$ transmite periodicamente beacons contendo sua identificação $(i d)$, posição atual (pos), número de vizinhos $(n V i z)$ e perfil social (profile $(v)$ ) para permitir o reconhecimento da comunidade. Quando um veículo $v_{i}$ recebe um beacon de um veículo $v_{j}$, ele verifica se profile $\left(v_{i}\right)==\operatorname{profile}\left(v_{j}\right)$. Se os perfis forem compatíveis, $v_{i}$ armazena $v_{j}$ na sua lista de vizinhos dispostos a cooperarem $\left(n b r \operatorname{List}\left(v_{i}\right)\right)$. Os beacons são constantemente trocados para auxiliar na manutenção da comunidade. O componente Influência Social trata da importância social que o nó atual tem na comunidade. Considerando a nbr List e métricas sociais, ele calcula quais vizinhos são mais propensos a cooperarem analisando os seus parâmetros sociais. Este trabalho utiliza a métrica Structural Influence (STR), que denota o quão um nó é influente na rede baseado na sua posição atual, sendo que quanto mais influente é um nó, maior a sua contribuição na eficácia da disseminação de dados [Nagaraj et al. 2019].

O módulo de Controle de Disseminação trata da detecção do evento, da seleção dos veículos que irão cooperar e da disseminação do evento até a EE. Ele possui os componentes Detecção e Seleção. A função do componente Detecção é detectar e iniciar o monitoramento de eventos. Quando um veículo $v d$ está no raio de alcance de um evento $e v, v d$ automaticamente assume o papel de Detector, coletando os dados de contexto de $e v$, que são: instante de tempo da detecção, localização, velocidade e direção. Por razões de simplificação, assumimos que a análise e detecção do evento são realizadas através de técnicas de processamento e análise de imagens obtidas por sensores de câmeras embarcadas nos veículos. Além disso, também assumimos que durante a análise do evento, o módulo Detecção estima o Time-to-Live (TTL). TTL é a quantidade máxima de vezes que uma mensagem deve ser repassada. Quando as informações de contexto do evento são obtidas, o componente Seleção inicia o processo de definir os outros dois papéis que os veículos podem assumir durante a cooperação à disseminação, que são: (i) Retransmissor: o veículo responsável por realizar a disseminação das informações na vizinhança, além de selecionar o próximo nó retransmissor; (ii) Gateway: veículo que entrega as informações do evento monitorado à EB e, consequentemente, à EE. Considerando o nó atual como o nó detector $v d$, a comunidade $c_{k}$ e que $v d \in c_{k}, v d$ considera como possíveis colaboradores os nós que fazem parte de $c_{k}$. Assim, $\forall v_{i} \in c_{k}$, se $v_{i}$ estiver ao alcance de uma EB, então $v_{i}$ é gateway. Se $v_{i}$ possuir a maior STR (métrica social) dentre todos os vizinhos de $v d$ que estão em $c_{k}$, então $v_{i}$ é retransmissor. Vale salientar que a seleção é 
iniciada tanto por $v d$ ao detectar um evento, quanto por qualquer $v_{i}$ que foi previamente selecionado como retransmisssor. Uma vez selecionado o retransmissor, $v d$ (ou vi) gera as mensagens de monitoramento e as dissemina na sua vizinhança. $\mathrm{O}$ nó também transmite o $i d$ do próximo retransmissor. Além disso, se $v d$ (ou $v i$ ) está ao alcance de uma EB, também entrega a informação à EB.

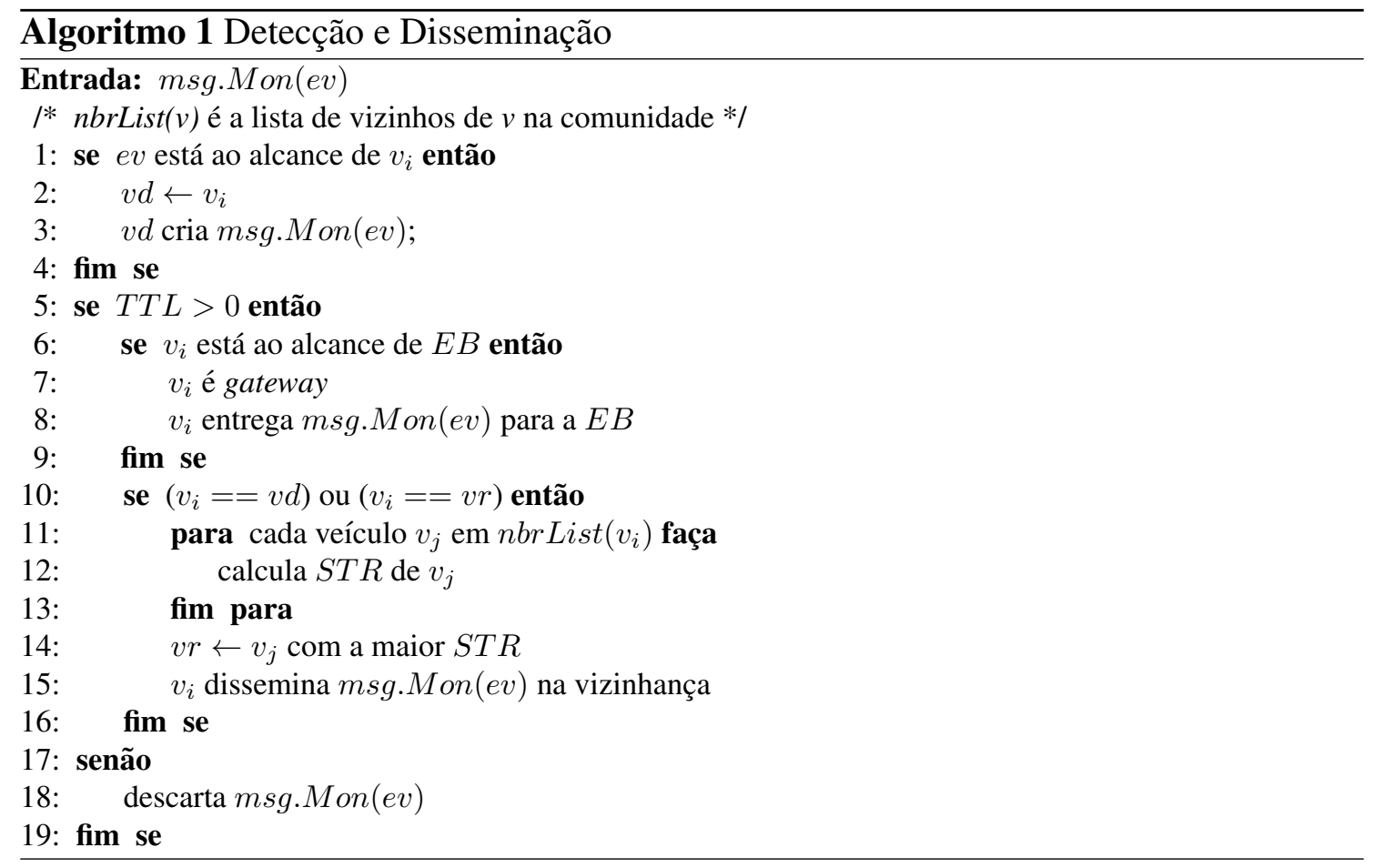

O Algoritmo 1 descreve a operação do SOCIABLE executada por um veículo $v_{i}$ ao detectar um evento $e v$ ou ao receber uma mensagem de monitoramento msg.Mon $(\mathrm{ev})$. Quando $v_{i}$ detecta $e v$, ele torna-se um veículo detector $v d$ e cria $m s g . M o n(e v)$ (linhas 1-4). Independentemente se $v_{i}$ recebeu ou criou msg.Mon $(e v), v_{i}$ verifica se o seu TTL ainda não expirou (linha 5). Caso o TTL tenha expirado, $v_{i}$ descarta a mensagem, e caso contrário, $v_{i}$ verifica se está ao alcance de uma EB. Se estiver, então $v_{i}$ é gateway, entregando assim a mensagem (linhas 6-8). Se $v_{i}$ é detector $v d$ ou é retransmissor $v r$ (linha 10), então $v_{i}$ inicia o reconhecimento da comunidade. No reconhecimento, $v_{i}$ verifica quais dos seus vizinhos possui a maior STR a fim de informá-lo que ele é o próximo retransmissor $v r$. Por fim, $v_{i}$ realiza a disseminação de $m s g . M o n(e v)$ (linhas 11-15).

\subsubsection{Estratégia de seleção de veículos retransmissores}

A seleção dos veículos retransmissores leva em conta os valores de QoS e QoE providos pelos veículos da comunidade. Os valores de QoE são baseados em valores sociais que os dispositivos oferecem, e neste caso emprega-se o SOR. O próximo veículo escolhido a ser o retransmissor trata-se do veículo vizinho com a maior STR, calculada conforme a Equação 1 [Nagaraj et al. 2019]. A STR considera quatro métricas de centralidade que são [Freeman 1978, Bonacich 1987]: Betweenness Centrality (BC), que corresponde a quantas vezes um nó está no menor caminho entre todos os pares de nós; Closeness Centrality $(C C)$, que indica o quão próximo um nó está de todos os outros nós; Degree Cen- 
trality $(D C)$, que corresponde a quantidade de vizinhos que o nó possui e; Eigenvector Centrality $(E C)$, que indica o número de nós importantes conectados ao nó atual. Neste trabalho, consideramos os nós importantes aqueles que estão ao alcance de EBs. Cada métrica possui um peso associado $(W)$, e a soma dos quatro pesos é 1 .

$$
S T R_{i}=W_{B C} *(B C)_{i}+W_{C C} *(C C)_{i}+W_{D C} *(D C)_{i}+W_{E C} *(E C)_{i}
$$

As três primeiras métricas melhoram o indicador de QoS já que ao escolher um veículo com valores melhores de $B C, C C$ e $D C$, aumenta-se a probabilidade de entrega de dados [Rahim et al. 2018a]. Por outro lado, a métrica $E C$ influencia o QoE uma vez que, se os dados alcançam um gateway o mais rápido possível (e consequentemente o usuário), a satisfação do usuário aumenta. Assim, quando um veículo detector seleciona o próximo retransmissor, ele valoriza mais QoS (valores maiores de $W_{B C}, W_{C C}$ e $W_{D C}$ e menos QoE (valor menor de $W_{E C}$ ). À medida que os próximos retransmissores são escolhidos a importância de QoS diminui enquanto a de QoE aumenta. A Figura 3 ilustra o processo de seleção, onde um veículo $v d$ detecta um evento e escolhe o próximo retransmissor utilizando um peso maior para QoS do que para QoE $\left(w_{p}>w_{q}\right)$. Conforme os próximos retransmissores são escolhidos, o valor de $w_{p}$ diminui enquanto $w_{q}$ aumenta. Este processo continua até que o pacote alcance o gateway.

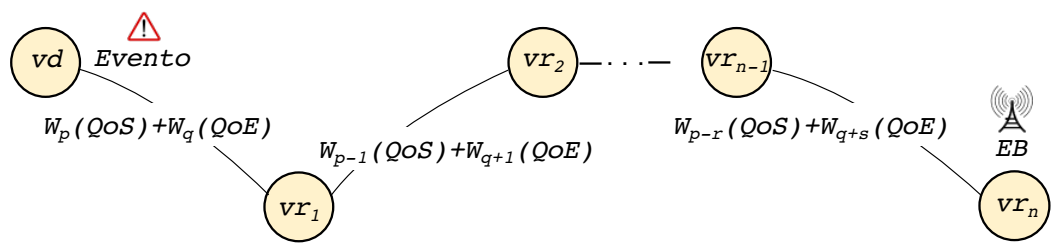

Figura 3. Seleção de retransmissor

\subsection{Funcionamento}

A Figura 4 exemplifica a operação do SOCIABLE ao detectar um evento urbano $e v$ ocorrendo em um instante de tempo. Neste exemplo, temos um cenário que corresponde à área de uma parte de duas vias de trânsito em duplo sentido composto pelo conjunto $V=\{v 1, v 2, v 3, v 4, v 5, v 6, v 7\}$ e uma estação base $E B$ de apoio a transmissão dos dados de eventos, neste caso o evento $e v$. Além disso, os veículos executando o SOCIABLE formam neste instante de tempo, duas comunidades $C 1$ e $C 2$, cores verde e azul, respectivamente. O veículo $v 1$ ao detectar $e v$ simultaneamente inicia o reconhecimento da comunidade e o monitoramento, criando assim o pacote $P$ com um $T T L=3$, que especifica o número de transmissões possíveis de $P$. Durante o reconhecimento da comunidade, $v 1$ constata que, dentre os seus vizinhos, apenas $v 3$ faz parte de sua comunidade $C 1$. Assim, independente do seu valor de $S T R, v 3$ é escolhido como o próximo retransmissor. Considerando que a cada transmissão de $P$, o seu $T T L$ diminui de uma unidade, quando $v 3$ recebe $P$, seu $T T L=2$, podendo então ainda retransmitir $P$.

Ao retransmitir $P, v 3$ descobre que possui dois vizinhos, $v 4$ e $v 6$, em $C 1$. Dentre eles, $v 3$ escolhe aquele que possui o maior valor de $S T R$, podendo assim alcançar mais nós na rede. Neste caso, $v 6 \mathrm{com} S T R=12$ é escolhido como retransmissor. Pelo exemplo, é possível notar que à medida que o pacote é retransmitido, o retransmissor aumenta o peso (influência) do indicador de QoE enquanto diminui o peso do indicador de 


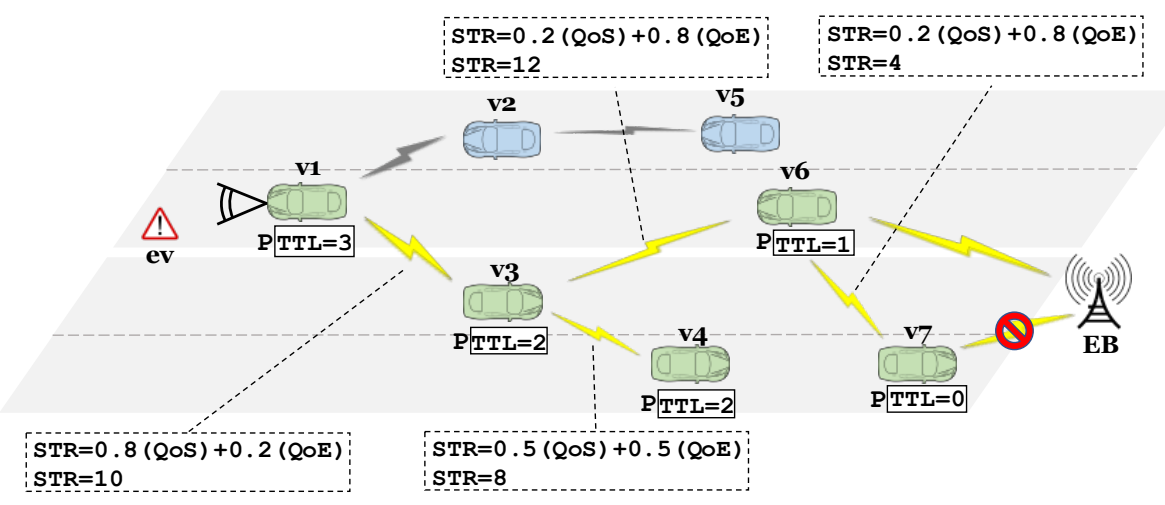

Figura 4. Exemplo de operação do SOCIABLE

QoS. Como $v 4$ possui um $S T R=8$, não é retransmissor, nem está ao alcance de uma EB, ele descarta $P$. Já $v 6$ está ao alcance de uma EB (gateway), e ele entrega $P$. Além disso, $v 6$ também é retransmissor, e ele repassa $P$ para $v 7$. Por fim, apesar de $v 7$ também estar ao alcance de uma EB, ele verifica que o TTL de $P$ expirou $(T T L=0)$, descartando assim a mensagem. Neste cenário exemplo, conforme os nós retransmissores são escolhidos, é criado o grupo $D(e v)=\{v 1, v 3, v 6, v 7\}$ que são os veículos que cooperaram para disseminar os dados do evento.

\section{Avaliação}

Esta seção apresenta a metodologia de implementação e a avaliação do sistema SOCIABLE para verificar a eficiência e robustez no monitoramento e disseminação de eventos críticos urbanos, bem como os aspectos sociais do sistema e sua influência e benefício à disseminação. O SOCIABLE foi implementado em C++ e testado no simulador NS3, versão 3.28, juntamente com o SUMO. Foi utilizado o projeto LuST (Luxembourg SUMO Traffic) como cenário de mobilidade de tráfego real ${ }^{1}$. As simulações foram realizadas em dois cenários distintos, considerando diferentes números de faixas na via de trânsito, sentido da via e número de veículos trafegando. Foi utilizado um único evento fixo com duração total de 8 minutos. Para a realização dos testes, consideramos comunidades formadas por veículos que possuem a mesma rotina. Ou seja, sendo um veículo $v$, um intervalo de tempo $t$ e uma área $a$, temos que $v(t, a)$ denota que o veículo $v$ está na área $a$ no intervalo $t$. Assim, dois veículos $v_{i}$ e $v_{j}$ criam um relacionamento se $v_{i}\left(t_{i}, a_{i}\right)==v_{j}\left(t_{j}, a_{j}\right)$. Assim sendo, utilizamos uma taxa de relacionamento entre veículos de 90\%. Ou seja, 90\% dos veículos dos cenários de teste fazem parte da comunidade. Apesar de ser uma taxa alta, ela pode ser alcançada se considerarmos os motoristas que trafegam em um trecho de via sempre no mesmo horário, como por exemplo, ao levar os filhos para a escola. O SOCIABLE foi comparado ao MINUET em termos de desempenho visto que o MINUET não apresenta características sociais.

A Figura 5 mostra os dois recortes do LuST empregados como cenários de alta densidade $(\mathrm{AD})$ e baixa densidade (BD), e as respectivas vias usadas na simulação. Os círculos amarelos correspondem às posições onde os eventos ocorrem nas vias. A Tabela 1 sumariza os parâmetros das simulações dos dois cenários. As métricas para aferir a eficiência do SOCIABLE e do MINUET foram: Número de Veículos Colaborando

\footnotetext{
${ }^{1}$ https://github.com/lcodeca/LuSTScenario
} 


\begin{tabular}{llll} 
Tarâmetro & BD & AD \\
\hline Tempo da simulação & 9 mins & 9 mins \\
Número de eventos & 1 & 1 \\
Tempo de duração do evento & 8 mins & 8 mins \\
Sentido da via & sentido único & duplo sentido \\
Número de faixas & 3 & 6 \\
Alcance de trans. veículos & $100 \mathrm{~m}$ & $100 \mathrm{~m}$ \\
Número de veículos & 91 & 754 \\
Número de estações-base & 1 & 1 \\
\hline
\end{tabular}

Figura 5. Cenários

Tabela 2. Métricas de desempenho e sociais aplicadas na avaliação

\begin{tabular}{|c|c|}
\hline Métrica & Descrição \\
\hline $\mathrm{N}^{\circ}$ de Veículos Colaborando (NVC) & $\begin{array}{l}\text { número de veículos que colaboram na disseminação (detector, retrans- } \\
\text { missor e gateway) ao longo do tempo }\end{array}$ \\
\hline Atraso Médio de Entrega (AME) & $\begin{array}{l}\text { intervalo de tempo entre o instante do evento detectado a primeira vez e } \\
\text { o instante que a primeira mensagem de monitoramento é entregue à EB }\end{array}$ \\
\hline $\begin{array}{l}\mathrm{N}^{\circ} \text { Pacotes Monitoramento Criados } \\
(\mathrm{NPMC})\end{array}$ & $\begin{array}{l}\text { quantidade de pacotes de monitoramento que foram criados pelos } \\
\text { veículos detectores }\end{array}$ \\
\hline $\begin{array}{l}\mathrm{N}^{\circ} \text { Pacotes Monitoramento Entre- } \\
\text { gues (NPME) }\end{array}$ & quantidade de pacotes de monitoramento que foram entregues à EB \\
\hline $\begin{array}{l}\text { Sobrecarga de Mensagens na Rede } \\
\text { (SMR) }\end{array}$ & $\begin{array}{l}\text { quantidade de pacotes transmitidos de monitoramento e beacons na rede } \\
\text { durante a simulação }\end{array}$ \\
\hline Influência Estrutural (STR) & corresponde ao quão influente um nó é na rede baseado na sua posição \\
\hline
\end{tabular}

(NVC), Atraso Médio de Entrega (AME), Número de Pacotes de Monitoramento Criados (NPMC), Número de Pacotes de Monitoramento Entregues (NPME), Sobrecarga de Mensagens na Rede (SMR). Na análise do comportamento social do SOCIABLE aplicouse a métrica Influência Estrutural dos Veículos (STR), que envolve outras quatro métricas sociais, como explicado na Seção 3.1.1. A Tabela 2 apresenta a descrição das métricas.

\subsection{Análise}

Esta seção apresenta as análises dos testes realizados para avaliar o desempenho do SOCIABLE comparado ao MINUET, além do comportamento social do SOCIABLE. Os gráficos da Figura 6 mostram a quantidade de veículos colaborando ao longo do tempo (NVC) nos cenários de alta (AD) e baixa densidade (BD). Diferente do MINUET que utiliza inundação, a estratégia empregada pelo SOCIABLE de disseminação direcionada resulta em uma quantidade menor de veículos colaborando (NVC menor) em ambos os cenários. No cenário BD (Gráfico 6(a)) o SOCIABLE permitiu a colaboração de no máximo 7 veículos e o MINUET de 12 veículos. No cenário AD (Gráfico 6(b)), 6 e 14 veículos colaboraram no SOCIABLE e no MINUET, respectivamente. Um NVC maior pode indicar super utilização de recursos, já que resultados semelhantes, ou até mesmo melhores, podem ser alcançados com menos veículos colaborando. Além disso, ao limitar a disseminação somente a membros da comunidade, o SOCIABLE previne o comportamento egoísta dos veículos. 

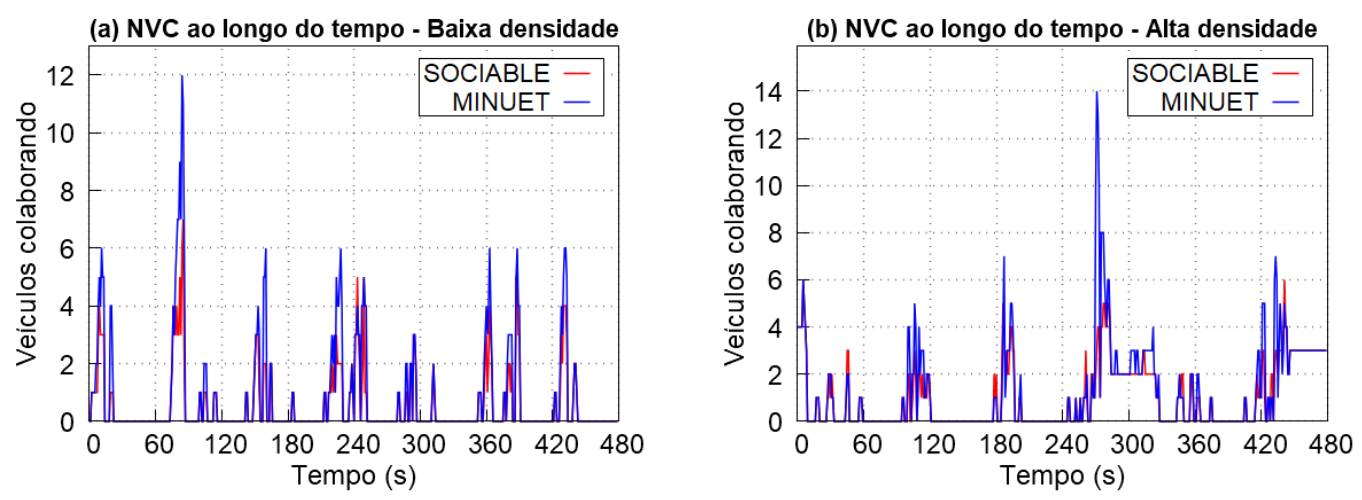

Figura 6. Veículos cooperando na disseminação em alta e baixa densidades

Os gráficos da Figura 7 exibem o atraso médio de entrega (AME) de pacotes de monitoramento gerados pelo número de veículos que colaboraram para a entrega. Em ambos os cenários $\mathrm{BD}$ e $\mathrm{AD}$ (Gráficos 7(a) e 7(b), respectivamente), uma quantidade menor de veículos colaboram no SOCIABLE para entregar os pacotes. No cenário BD observa-se uma tendência de subida do AME no SOCIABLE e no MINUET de até 7 veículos, sendo que o SOCIABLE obteve um pico de atraso 35,26\% maior que o pico do MINUET, o que ainda é um atraso adequado para transmissões em tempo real. No cenário $\mathrm{AD}$ os dois sistemas tiveram valores próximos. A partir de 7 veículos, não há mais entrega de pacotes pelo SOCIABLE, enquanto que o AME do MINUET estabiliza. Estes resultados demonstram que o SOCIABLE consegue entregar pacotes em tempo real e maior eficiência no uso dos recursos (quantidade menor de veículos para entregar pacotes), possibilitando assim boa satisfação ao usuário $(\mathrm{QoE})$ ao mesmo tempo que apresenta boa Qualidade de Serviço (QoS).
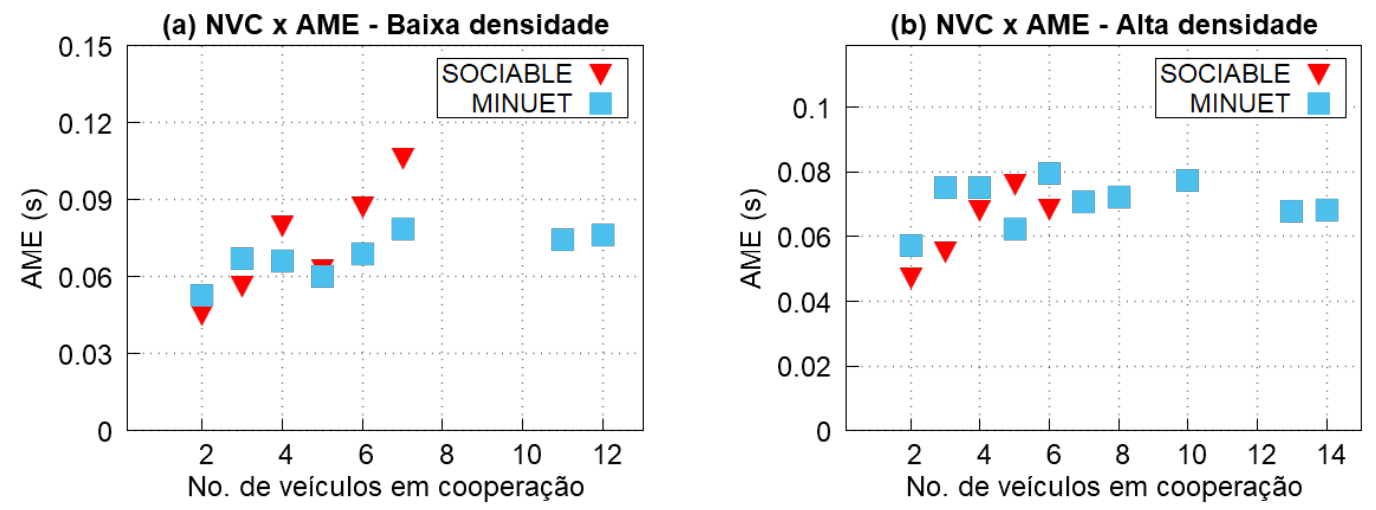

Figura 7. Atraso médio de entrega de mensagens sob alta e baixa densidades

Os gráficos da Figura 8 apresentam a quantidade de pacotes de monitoramento criados (NPMC) quando o evento é detectado e de pacotes entregues (NPME) à EB de acordo com o número de veículos colaborando (NVC) no SOCIALBLE e no MINUET. Em ambos os cenários (Gráficos 8(a) e (b)), o SOCIABLE obteve NPMC igual ao do MINUET mesmo com uma quantidade menor de veículos colaborando. Isto acontece porque há o mesmo número de veículos detectores. No cenário $\mathrm{BD}$, o SOCIABLE alcançou um NPME maior que o MINUET quando há menos veículos colaborando. À medida que mais veículos colaboram, o MINUET obteve um NPME maior. No cenário AD, o SO- 
(a) NVC x NPMC / NPME - Baixa densidade

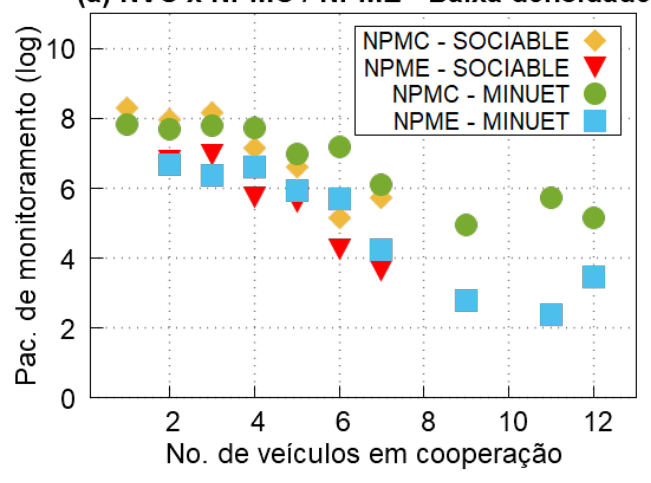

(b) NVC x NPMC / NPME - Alta densidade

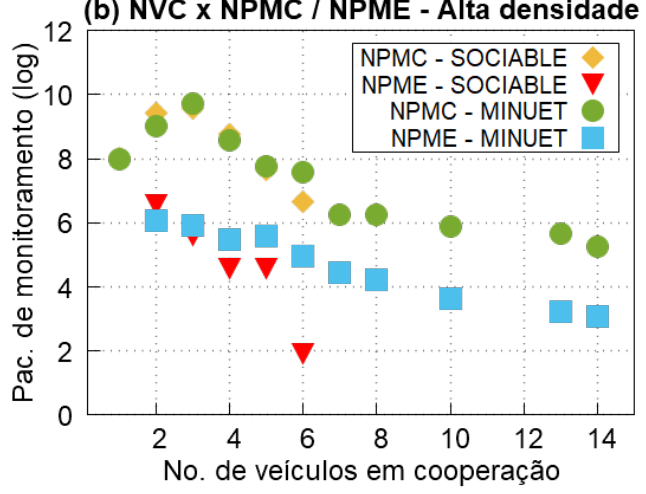

Figura 8. Qtde de pacotes criados e entregues à EB por ambos os sistemas

CIABLE alcançou um NPME maior do que o MINUET apenas quando dois veículos colaboram. Ainda neste cenário, o MINUET conseguiu entregar pacotes com até catorze veículos colaborando. O MINUET sempre possui mais veículos colaborando por empregar a técnica de inundação. Como o SOCIABLE utiliza parâmetros sociais ao escolher o próximo retransmissor, apenas um é escolhido a cada repasse, diminuindo o número de cópias de pacotes trafegando na rede. Estes resultados indicam que o SOCIABLE e o MINUET conseguem detectar, monitorar e entregar pacotes de monitoramento de eventos urbanos à EB em diferentes condições de ambiente. Entretanto, uma quantidade menor de veículos colaboram para a entrega destes pacotes no SOCIABLE, o que mostra a sua maior eficiência no uso de recursos.

(a) SMR - Baixa densidade

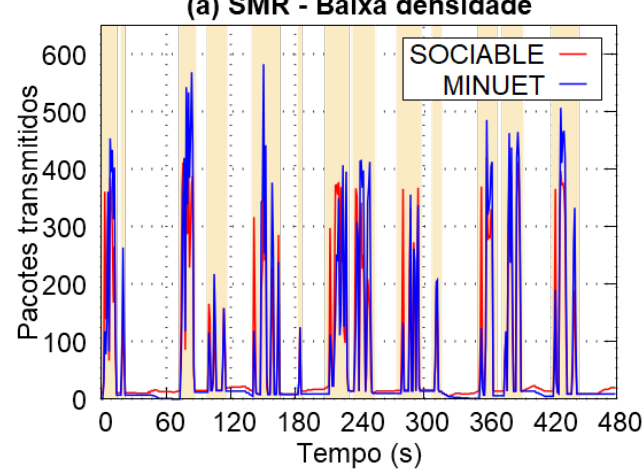

(b) SMR - Alta densidade

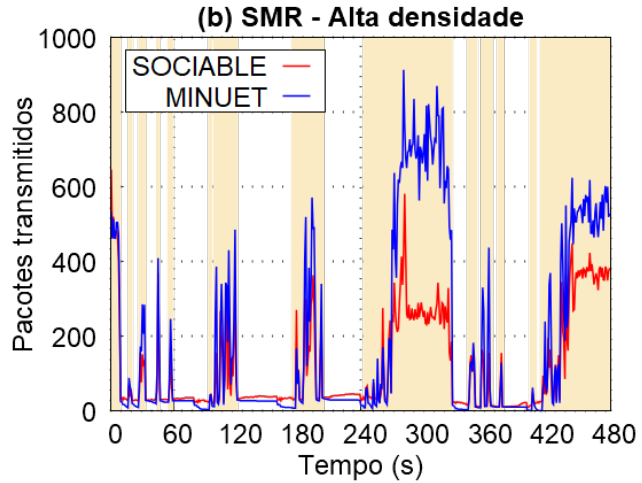

Figura 9. Custo de comunicação sob alta e baixa densidades

Os gráficos da Figura 9 mostram o custo de comunicação (SMR) do SOCIABLE e do MINUET nos cenários com alta e baixa densidades. Devido ao repasse direcionado empregado pelo SOCIABLE, os seus valores de SMR são menores, alcançando taxas de $3,49 \%$ e $36,56 \%$ a menos de mensagens transmitidas na rede nos cenários $\mathrm{BD}$ e $\mathrm{AD}$, respectivamente. Este resultado, em conjunto com os resultados anteriores demonstram como os parâmetros sociais do SOCIABLE auxiliam num melhor uso dos recursos da rede em relação ao MINUET, ao mesmo tempo que consegue realizar o monitoramento, a disseminação e a entrega de pacotes.

A Figura 10 mostra a influência das métricas de centralidade sobre as métricas AME (Gráfico 10(a)) e NPME (Gráfico 10(b)). Os valores do eixo x dos gráficos ex- 
pressam o intervalo do valor normalizado de STR. No Gráfico 10(a), o cenário de alta densidade tem um menor AME porque ao selecionar veículos retransmissores com maiores valores de centralidade (BC, CC, DC e EC) aumentou-se as chances de selecionar retransmissores melhor localizados na rede e próximos à EB. Por outro lado, no Gráfico 10(b) no cenário de baixa densidade, SOCIABLE entrega mais pacotes de monitoramento (NPME maior) uma vez que os veículos passam mais tempo monitorando o evento. Esses resultados mostram que o comportamento social afeta o desempenho do sistema. Apesar disso, o SOCIABLE entrega dados do evento sob diferentes condições sociais.

(a) AME x STR

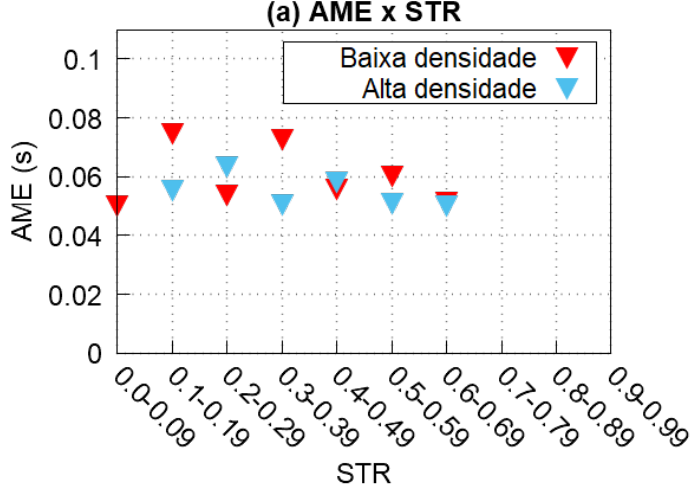

(b) NPME x STR

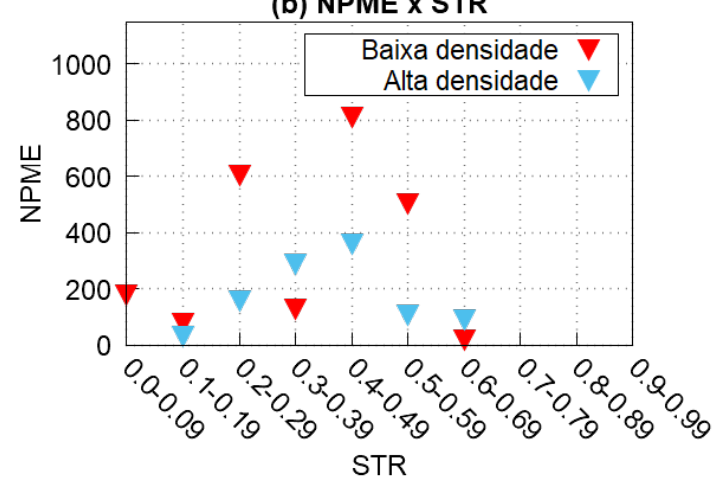

Figura 10. Influência social no atraso e na entrega de pacotes

\section{Conclusões}

Este trabalho apresentou o sistema SOCIABLE para auxiliar na disseminação de eventos críticos urbanos em ambientes SIoV. Ele consegue entregar dados de eventos urbanos às entidades externas utilizando comunidades criadas a partir dos relacionamentos sociais entre os veículos. Resultados obtidos por simulações demonstram que o SOCIABLE consegue escolher os veículos cooperativos que participarão na disseminação do evento e entregar as mensagens à estação base em tempo real. Além disso, ele consegue disseminar de forma robusta ao longo de toda a ocorrência do evento. Comparações feitas com uma solução sem parâmetros sociais mostram que o SOCIABLE alcança resultados semelhantes ou melhores com uma sobrecarga menor da rede. Como trabalhos futuros, pretendemos analisar o desempenho do SOCIABLE diante de eventos sobrepostos no tempo e espaço, além de considerar outros mecanismos à formação das comunidades.

\section{Referências}

Akabane, A. T., Immich, R., Madeira, E. R., and Villas, L. A. (2019). IMOB: An Intelligent Urban Mobility Management System Based on Vehicular Social Networks. In IEEE Vehicular Networking Conference, VNC, volume 2018-Decem.

Andrade, J. E., Veloso, K., Silva, N. d. V., dos Santos, A. L., and Matos, F. M. (2019). Monitoramento e Disseminação Cooperativa de Eventos Emergenciais Apoiado por Agrupamentos de Veículos. In Anais do Workshop de Gerência e Operação de Redes e Serviços, pages 127-140.

Bonacich, P. (1987). Power and centrality: A family of measures. American Journal of Sociology, 92(5):1170-1182.

Campolo, C., Molinaro, A., and Iera, A. (2018). A reference framework for social-enhanced Vehicle-to-Everything communications in 5G scenarios. Computer Networks, 143:140-152. 
Cunha, F. D., Maia, G., Viana, A. C., Mini, R. A., Villas, L. A., and Loureiro, A. A. (2014). Socially inspired data dissemination for Vehicular Ad Hoc Networks. MSWiM 2014 - Proceedings of the 17th ACM International Conference on Modeling, Analysis and Simulation of Wireless and Mobile Systems, pages 81-85.

Freeman, L. C. (1978). Centrality in social networks conceptual clarification. Social Networks, $1(3): 215-239$.

Khan, S. and Franzle, M. (2015). Robust mid-range communication in Urban VANETs. In International Conference on Advanced Communication Technology, ICACT, pages 115-120.

Monreal, C. O. (2018). Future urban mobility group: Smart cities research institute swinburne university of technology, Melbourne Australia. IEEE Intelligent Transportation Systems Magazine, 10(2):203-205.

Nagaraj, K., Bhasale, S. S., McNair, J., and Helmy, A. (2019). Vulnerability assessment and classification based on influence metrics in mobile social networks. In Proceedings of the 17th ACM International Symposium on Mobility Management and Wireless Access, MobiWac '19, page 9-16, New York, NY, USA. Association for Computing Machinery.

Rahim, A., Kong, X., Xia, F., Ning, Z., Ullah, N., Wang, J., and Das, S. K. (2018a). Vehicular Social Networks: A survey. Pervasive and Mobile Computing, 43:96-113.

Rahim, A., Ma, K., Zhao, W., Tolba, A., Al-Makhadmeh, Z., and Xia, F. (2018b). Cooperative data forwarding based on crowdsourcing in vehicular social networks. Pervasive and Mobile Computing, 51:43-55.

Shrestha, R., Bajracharya, R., and Nam, S. Y. (2018). Centralized approach for trustworthy message dissemination in VANET. In IEEE/IFIP Network Operations and Management Symposium: Cognitive Management in a Cyber World, NOMS 2018, pages 1-5.

Silva, R. and Iqbal, R. (2019). Ethical Implications of Social Internet of Vehicles Systems. IEEE Internet of Things Journal, 6(1):517-531.

Vegni, A. M. and Loscrí, V. (2015). A survey on vehicular social networks. IEEE Communications Surveys and Tutorials, 17(4):2397-2419.

Wang, X., Ning, Z., Hu, X., Ngai, E. C., Wang, L., Hu, B., and Kwok, R. Y. (2018). A City-Wide Real-Time Traffic Management System: Enabling Crowdsensing in Social Internet of Vehicles. IEEE Communications Magazine, 56(9):19-25.

Wang, X., Ning, Z., Zhou, M. C., Hu, X., Wang, L., Zhang, Y., Yu, F. R., and Hu, B. (2019). Privacy-Preserving Content Dissemination for Vehicular Social Networks: Challenges and Solutions. IEEE Communications Surveys and Tutorials, 21(2):1314-1345.

Wu, F.-J. and Solmaz, G. (2019). CrowdEstimator: Approximating Crowd Sizes with Multimodal Data for Internet-of-Things Services. In MobiSys 2018 - Proceedings of the 16th ACM International Conference on Mobile Systems, Applications, and Services, pages 337-349.

Xu, W., Guo, S., Zhou, H., Ma, S., and Wu, M. (2019). A Queueing Analysis of the Opportunistic Vehicle-to-Vehicle Communication. In IEEE Global Comm. Conference, pages 25-30.

Yaqub, M. A., Ahmed, S. H., Bouk, S. H., and Kim, D. (2018). Enabling critical content dissemination in vehicular named data networks. In Proceedings of the 2018 Research in Adaptive and Convergent Systems, RACS 2018, pages 94-99. Association for Computing Machinery, Inc. 